\title{
A Framework for Motivating Sketching Practice with Sketch-based Gameplay
}

\author{
Blake Williford \\ Sketch Recognition Lab \\ Texas A\&M University \\ bwilliford@gmail.com
}

\author{
Wayne Li \\ College of Design \\ Georgia Tech \\ wayne.li@design.gatech.edu
}

\author{
Matthew Runyon \\ Sketch Recognition Lab \\ Texas A\&M University \\ mattrunyon@tamu.edu
}

\author{
Julie Linsey \\ iDREEM Lab \\ Georgia Tech \\ julie.linsey@me.gatech.edu
}

\author{
Josh Cherian \\ Sketch Recognition Lab \\ Texas A\&M University \\ jcherian14@tamu.edu
}

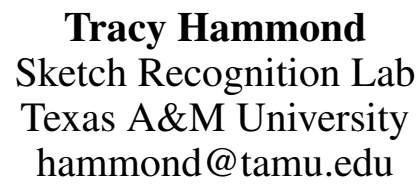

\begin{abstract}
Sketching is a valuable skill to learn but requires extensive motivation and practice to improve. We present a framework for motivating practice with sketch-based gameplay that is rooted in a grounded theory study of the motivations of various individuals with different skills levels. The individuals interviewed included a range from novice and intermediate industrial design students to established design professionals. Four categories emerged that explain the differences in motivation between individuals with different skill levels, including achievement, competition, communication, and creativity. We also present a case study of the implementation of two different gameplay approaches for encouraging line work practice in a high school art course and a university sketching course. The study revealed that both approaches were very engaging and motivating to students, with 72,842 lines practiced across the 150 students overall. We also gained insights about how the approaches differed in motivating students, and share principles we learned on motivating students with gameplay that may be useful to other researchers, educators, and technologists.
\end{abstract}

\section{Author Keywords}

Sketching; Drawing; Art Education; Design Education; Gameplay; HCI; Human-computer Interaction; Games; Serious games; Game-based Learning; Gamification; Motivation

\section{CCS Concepts}

-Human-centered computing $\rightarrow$ Interactive systems and tools; Interaction techniques; •Applied computing $\rightarrow$ Computer-assisted instruction;

Permission to make digital or hard copies of all or part of this work for personal or classroom use is granted without fee provided that copies are not made or distributed for profit or commercial advantage and that copies bear this notice and the full citation on the first page. Copyrights for components of this work owned by others than ACM must be honored. Abstracting with credit is permitted. To copy otherwise, or republish, to post on servers or to redistribute to lists, requires prior specific permission and/or a fee. Request permissions from permissions@acm.org.

CHI PLAY'19, October 22-25, 2019, Barcelona, Spain.

Copyright (C) 2019 Association of Computing Machinery.

ACM ISBN 978-1-4503-6688-5/19/10 ...\$15.00.

http://dx.doi.org/10.1145/3311350.XXXXX

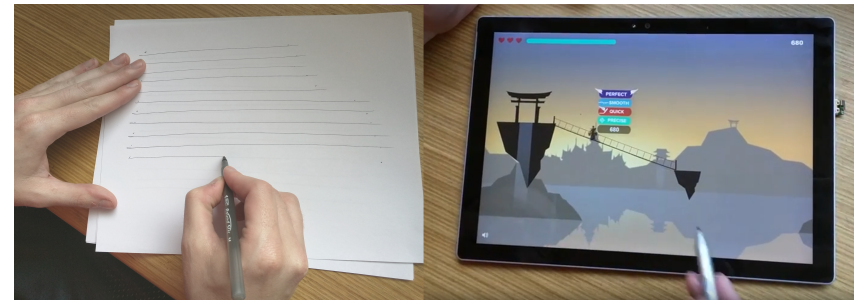

Figure 1. Sketch-based gameplay can help motivate practice of sketching fundamentals. We developed a theoretical framework for motivating individuals of varying skill levels, and explored two different gameplay approaches to a popular connect-the-dots exercise for practicing line work. The left image shows the traditional exercise with pen and paper, while the right image shows one of our gameplay approaches ZenSketch.

\section{INTRODUCTION}

Sketching has traditionally been considered a valuable skill for professions such as industrial design, architecture, and engineering as it has been shown to benefit problem solving ability [39] and improve communication skills [7]. It is also increasingly valued in the multidisciplinary field of humancomputer interaction (HCI) as a means to rapidly generate ideas for user experiences [4, 26, 42]. However, the skill relies on many different fine motor skills and cognitive skills working together $[45,46]$ and it can take an individual years of practice to make improvements.

It is not uncommon to find people who lack confidence in their sketching ability, and a common remark one might hear is "I can't even draw a straight line!" This form of low self-efficacy [1] and learned helplessness is common in many people and often begins in childhood [12] as many children abandon artistic and creative activities. This low self-efficacy naturally makes it difficult to be motivated to practice. Even students studying industrial design and learning to sketch at a professional level struggle with motivation and can view practicing fundamentals as a "chore" [20].

Gamification, game-based learning, and serious games are promising areas of research which may help to motivate students in educational contexts. Serious games have shown to be more effective than traditional educational methods [54], but not necessarily more motivating. Ongoing research in these 
areas is exploring the degree to which games can motivate people and in what manner [18, 27, 50, 28]. There has even been exploration in to a comprehensive design framework for gamification in order to push the emerging field towards "inspiring and empowering" gameplay versus the more sinister "manipulative and obsessive" gameplay [6].

Our goal with this research was driven by two main research questions:

R1-What motivates individuals to practice sketching and how do those motivations change with skill level?

R2_-How are our current approaches to sketch-based gameplay motivating students?

To answer R1, we conducted a grounded theory study with eighteen participants, including four professionals. To answer $\mathrm{R} 2$, we conducted a semester-long implementation of two previously designed approaches to motivating line work practice with sketch-based gameplay in a university sketching course as well as a high school art course.

Our contributions in this work include:

- An analysis of how motivations of individuals with different sketching skill levels change and evolve. This analysis may be useful for other researchers, educators, and technologists involved in design education and/or art education.

- A framework for how various approaches to sketch-based gameplay can target these motivations and encourage sketching practice for individuals with different skill levels.

- The results of a semester-long case study of two sketchbased gameplay implementations in a university sketching course and a high school art course. We gathered interesting insights about how students engaged with our approaches and how they were motivated.

\section{RELATED WORK}

We initially describe some related sketch-based games and applications before diving in to other work in motivation theory and motivating students with games. We acknowledge that what constitutes a "game" and what does not can be quite subjective, but we borrow reasoning from Stenros [41] to define games as culturally-constructed artifacts.

\section{Sketch-based Games}

Sketch-based games are by no means a new concept. Casual classics like Tic-Tac-Toe and Hangman can be played with readily accessible pen and paper. Pictionary [34], a charadesinspired word guessing game that involves drawing, remains a popular board game decades after its creation.

With digital games now a prevailing medium, new sketchbased games have been created which utilize either finger swiping or the use of a digital stylus. DrawSomething [30], a mobile game that is very similiar to Pictionary is worth noting for how it promotes communication skill through sketching.

Other sketch-based games have been more oriented towards education. Many have been geared towards children, e.g., the work of Paulson et al. in encouraging learning through more tactile and kinesthetic approaches [31]. EasySketch2 was developed to assess and improve fine motor skills ion children in order to see how prepared they are for school [21]. TAYouKi $[48,47]$ was oriented towards teaching young children to draw basic shapes. A system developed by Truong et al. [44] helps children learn new concepts through sketch-based interaction. Wasson et al. [49] showed games can be utilized to learn art history and other general pictures.

Some games have been more oriented towards research and collecting data. PicturePhone and StellaSketch are collaborative sketch-based games that utilize human computation [24] to collect and classify sketch data [19]. Google released a game called QuickDraw that could guess what users were drawing using machine learning, and was meant to build a massive dataset of over 50 million doodles. These games offer fun play experiences for users while also fulfilling secondary research motives.

\section{Sketch-based Applications}

It is worthwhile to mention the many sketch-based tutorial applications that have been developed for teaching sketching skills. While they are not games, they offer interesting insights in digital approaches for improving sketching skills in people, and can inspire ways to incorporate gameplay.

Sketch Sketch Revolution [11], was a guided tutorial system for sketching that offers feedback on stroke speed and accuracy. The key insight from this work was to treat each line of a drawing as a granular element, and to offer the user a means to produce the same drawing as the tutorial creator line by line. It is not difficult to see how gameplay could be incorporated in to this as each line can serve as a core "loop" for a game.

PortraitSketch [55], ShadowDraw [25], and DrawMyPhoto [52] are tutorial systems based on computerassisted tracing. While tracing is useful for beginners to build confidence in drawing, it is not often used as a genuine tool for improvement in pedagogy of teaching drawing and sketching [22, 35]. How2Sketch [17] is a more recent work that can generate step-by-step sketching tutorials from 3D Models, however it does not provide real-time feedback on performance.

ICanDraw [8] is a guided tutorial system for sketching human faces that utilizes sketch recognition and template-matching to guide the user in properly proportioning faces as they draw them. ICanDraw is notable for how well it encourages accuracy, an important aspect of quality drawings, and particularly important for the nuances of the human face.

Aalto Drawing is an educational tool that shows promise for "fair and encouraging" automatic accuracy feedback on a variety of basic forms [23]. While it is limited to a star rating, and has yet to be formally evaluated, it hints at the possibility of the gamification of drawing practice and its potential to motivate students.

\section{Motivation Research}

Since this work is focused on motivating individuals, it's worth mentioning some sensitizing concepts in motivation theory that are relevant. Self-Determination Theory (SDT) [37] is one 
of the prevailing paradigms in psychology, as it describes people's innate psychological needs for competence, autonomy, and relatedness. When learning a new skill, individuals tend to be more extrinsically motivated at first, looking for outside rewards such as positive feedback or monetary gain. However through the natural process of self-determination, motivations are internalized and the individual starts to become more intrinsically motivated to learn the skill. Motivation in games has been widely studied and remains an interesting area of research since games have the potential to be very immersive and engaging. Richard Bartle [2] created a taxonomy of player types for massively-multiplayer online (MMO) games that describes their motivations. We draw inspiration from this 2-axis approach to motivations.

The work of Phillips et al. [32] explored how different in-game rewards motivate people. They found little difference between individual isolated rewards, but found that a greater variety of rewards has a positive impact on interest and enjoyment. This is an important insight in to the design of serious games to motivate students in educational contexts.

Serious games have been shown to be more effective than traditional educational methods in terms of learning and retention, [54] but not necessarily motivation. Ongoing research in game-based learning is exploring the degree to which games can motivate students more $[18,27,50]$.

\section{MOTIVATION STUDY}

In an effort to understand what motivates students to practice their sketching ability, we initially conducted a grounded theory study [5] with eighteen participants, including fourteen undergraduate and graduate industrial design students and four established industrial design professionals. Seven of the students were male, while seven were female. Figure 2 is a table that provides more detail on the participants.

Semi-structured interviews were conducted with these individuals with a primary focus on understanding what motivates them to practice sketching, and what factors hinder their motivation or discourage them. Interviews lasted approximately 20-30 minutes. Theoretical sampling [14] was used to ensure a variety of skill levels were represented as well as both males and females. The constant comparative method [13] was used to continuously update codes and categories as interviews were conducted.

Participants were asked to self-identify as being either Novice, Intermediate, or Advanced at sketching. Descriptions of these categories are below:

Novice-I am still learning fundamentals and have much to improve on. I am not very confident in my sketching ability.

Intermediate - I have mastered some fundamentals, but am still improving and learning. I am somewhat confident in my sketching ability.

Advanced-I have mastered fundamentals and advanced methods and am very experienced and confident in my sketching ability.

\begin{tabular}{|lcll}
\hline \multicolumn{4}{c}{ STUDENTS } \\
\hline CODE & GENDER & AGE & SKILL LEVEL \\
\hline S1 & Female & 25 & Novice \\
\hline S2 & Male & 24 & Advanced \\
\hline S3 & Female & 24 & Intermediate \\
\hline S4 & Male & 25 & Intermediate \\
\hline S5 & Male & 25 & Novice \\
\hline S6 & Male & 29 & Intermediate \\
\hline S7 & Male & 21 & Intermediate \\
\hline S8 & Female & 25 & Novice \\
\hline S9 & Female & 28 & Novice \\
\hline S10 & Female & 23 & Novice \\
\hline S11 & Male & 26 & Novice \\
\hline S12 & Female & 21 & Intermediate \\
\hline S13 & Female & 25 & Advanced \\
S14 & Male & 22 & Novice \\
\hline
\end{tabular}

\begin{tabular}{lccl}
\hline \multicolumn{4}{c}{ PROFESSIONALS } \\
\hline CODE & GENDER & AGE & SKILL LEVEL \\
\hline P1 & Male & 41 & Intermediate \\
P2 & Male & 42 & Advanced \\
P3 & Male & 24 & Intermediate \\
P4 & Male & 25 & Advanced \\
\hline
\end{tabular}

Figure 2. A Table showing participants interviewed during our grounded theory study.

Ultimately, four categories emerged which encompass what motivates students to practice their sketching ability: Achievement, Competition, Communication, and Creativity. A high level overview of the categories can be seen in Figure 3. More detail about these categories is described in the following sections.

\section{Achievement}

Particularly prominent among the most novice students interviewed, a sense of achievement was a strong motivator for practicing sketching. This was the most extrinsic and egodriven motivator. Some students wanted to just "feel" better at sketching, while others were looking for external praise from their instructor or peers. Some were simply driven to avoid negative criticism from their instructor or peers.

"I've never felt too motivated because I never get any praise from my teacher." - S10 

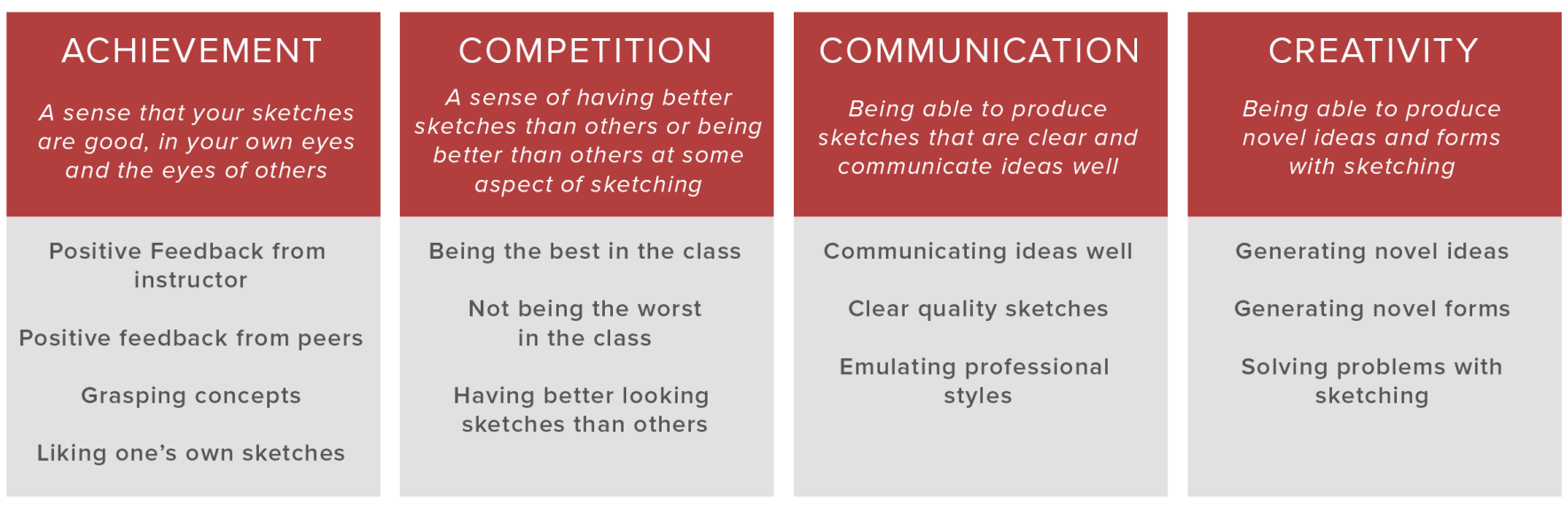

Figure 3. The four categories that emerged from our grounded theory analysis, along with high-level codes that contributed to the categories.

\section{"I'm afraid of my instructor criticizing me. I want to do well." -S1}

\section{Competition}

Some students expressed a desire to be the best in the class, or to have their sketches highlighted during critiques. Others simply wanted to avoid being the worst in the class. Such motivations fall under the category of competition. There was also a consensus that if there was going to be competition, everyone should be around the same level.

\section{"Competition can definitely motivate me, but only if they are around my skill level.”-S3}

"Some friendly competition and seeing my friend's sketches was always a motivator for me when I was a student."-P1

\section{Communication}

Some students were focused on the practical reasons for learning sketching such as collaborating and communicating ideas. These students tended to be more intermediate or advanced and were focused on their careers and finding jobs. From that perspective, the students were motivated by producing higher quality drawings that communicated ideas better and could be better understood by their peers.

\section{"If you have an idea and you can't put it on paper, it doesn't count."-S6 \\ "I'm always inspired to practice by seeing work from the pros like Syd Mead and Joe Johnston.”-P1}

\section{Creativity}

Some of the most advanced students and the professionals we interviewed were motivated by novelty and a desire to make the most creative ideas with sketching. The students who were most motivated by this were also approaching graduation and were focused on their careers. Since sketching and the rapid generation of ideas is a key part of the discipline of industrial design, it's no surprise that these individuals were motivated by creativity.
Interestingly, we found that the most advanced individuals also rejected competition as a motivator, with some even suggesting it can be a negative. This suggests a shift towards intrinsic mastery-driven motivation versus extrinsic ego-driven motivation. This is an ideal evolution of motivation from the standpoint of SDT [37].

\section{"Competition is not what sketching should be about."- P4 \\ "I've been in a position where others are discouraged because of my sketching ability, so I think it should be more about personal mastery." $-S 2$}

\section{MOTIVATION FRAMEWORK}

A fundamental insight of the grounded theory study was the realization that individual's motivations evolve based on skill level and confidence. Figure 4 shows an approximation of how individual's motivations change with skill level in a radar chart inspired by the gameplay motivation work of Richard Bartle [2], among others. We produced the charts by coding instances of each category for each participant and averaging them. While not a rigorous approach, it provides a general sense of these changes in motivation.

This insight influences a key component of our motivation framework - To employ different motivators based on an individual's self-efficacy and skill level, be it self-evaluated or determined by an intelligent system based on performance. The following sections explain this approach for novice, intermediate, and advanced individuals.

\section{Novice}

It is important for many novices to have a sense of achievement, providing a boost in self-efficacy and motivation that can carry over in to more time spent practicing. Achievement in the context of sketch-based gameplay can include high scores, RPG-influenced stat and skill improvements, improvements in performance metrics, and completed artifacts that evoke a sense of pride. Previous work has shown that when individuals invest time and effort in to creating something, it holds more value to them [29]. We believe it is best for novices to compete 

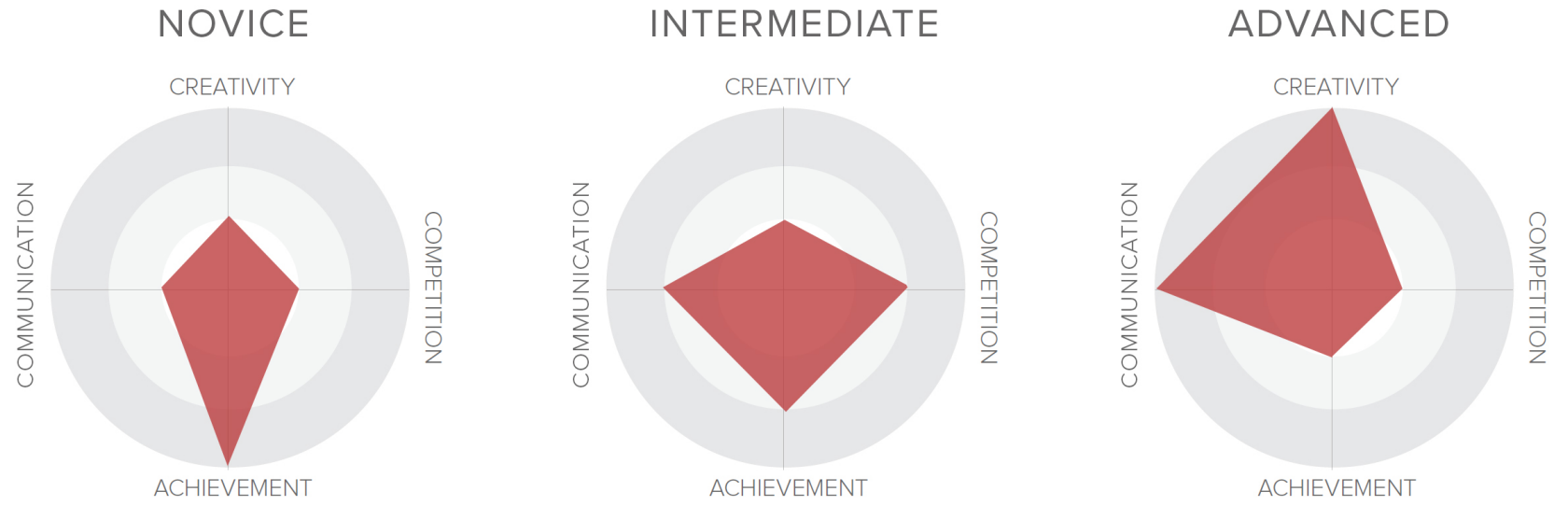

Figure 4. These radar charts depict an approximation of how motivations evolve as one's skill level increases in sketching. Naturally, not all individuals follow this exact evolution, but it was the general trend we found. Novices are primarily in need of a sense of achievement. As they become intermediate, their motivations can expand to competition and communication. Eventually, as they master sketching and become professionals, they see sketching as a useful tool, and are more motivated by creativity and communication, while being less interested in ego-driven motivations like achievement and competition. This follows self-determination theory.

with themselves initially, rather than competing with peers, since many individuals expressed being discouraged when compared to peers.

Recommended motivators for Novice skill level:

- Single-player gameplay

- High scores

- Achievements / Badges

- RPG-inspired leveling / stats

- Assistance in producing quality artifacts

\section{Intermediate}

At the intermediate level, motivators can expand to include competition and communication. At this point, individuals may have developed enough sketching self-efficacy to no longer be discouraged by comparison to peers.

Friendly competition can bring out strong motivation in individuals, particularly those who already identify as being competitive. Some individuals may not identify as being competitive at all, so we believe competition should always be an option rather than a requirement. Competition in the context of sketch-based gameplay can include leaderboards and thematic sketching competitions.

Communication can also be a strong motivator at this stage because individuals may be starting to understand that sketching is a useful and practical "tool." Pictionary and DrawSomething are examples of how gameplay can foster communication skills and possibly motivate someone to improve their sketching ability, although the games are not explicitly designed for that purpose. Nevertheless, the more clearly an individual can communicate an idea, the better they can perform in such games.

We recommended introducing these motivators for Intermediate skill level:
- Multiplayer gameplay

- Themed sketching competitions

- Collaborative gameplay

- Leaderboards

- Communication game mechanics

\section{Advanced}

As individuals become more advanced at sketching, they begin to tap in to their creativity and be highly motivated by producing truly novel ideas and forms. At this point it can be advantageous to use creativity as a motivator. Creativity in the context of sketch-based gameplay can include idea generation game mechanics, puzzles that require divergent thinking, etc.

It's also important to consider that at an advanced level, individuals can inspire others with their sketches and those that we interviewed expressed they were motivated to do so. It may be beneficial to bring in community aspects, and give these individuals the opportunity to both share their sketches and teach others concepts.

We recommended introducing these motivators for the Advanced skill level:

- Idea generation game mechanics

- Opportunities to share sketches

- Opportunities to teach others

\section{Limitations}

We recognize that the proposed four-component motivation model and Figure 4 are based on an exploratory qualitative analysis with students from one university industrial design program along with some associated professionals, and should be validated in future work using quantitative methodologies. We believe that the model does provide a general sense of the motivations of individuals with different skill levels learning 


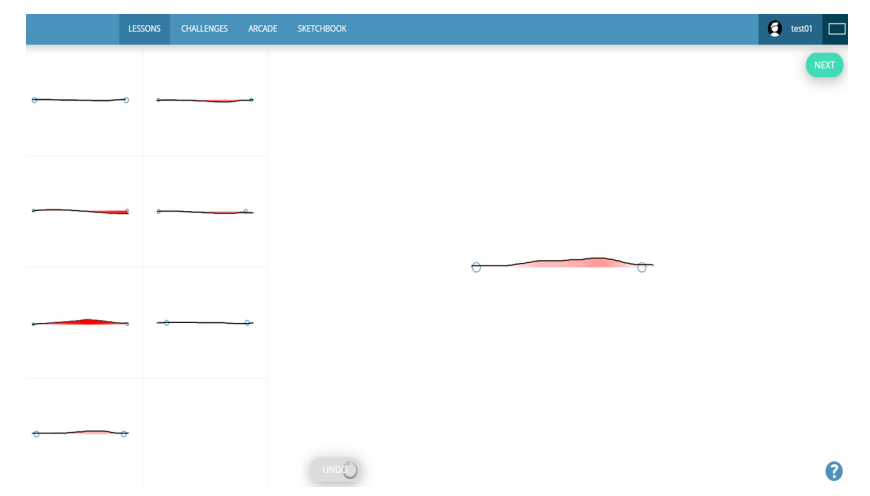

Figure 5. Screenshot of the gamified line lesson. The lesson provides some basic feedback on accuracy and a summative feedback screen, but is otherwise just a digital version of the classic connect-the-dots exercise.

sketching, however it could be improved and expanded upon with more quantitative methods such as as motivation scales [33] and more rigorous studies.

\section{CASE STUDY}

We conducted a study with students who were all novice to intermediate skill levels with respect to sketching, and explored two of the core motivators of our motivation framework: Achievement and competition. We focused mostly on understanding how these motivators influenced the students, and how much they encouraged more practice. We also wanted to compare two different approaches to sketch-based gameplay for basic line work-A gamified line lesson and a serious game for practicing line work called ZenSketch. Both are web-based prototypes that can be used on any device with an internet connection, and are described in more detail below as well as in the companion video.

\section{Gamified Line lessons}

The gamified line lesson is based on a popular exercise in design sketching pedagogy which involves connecting dots to improve freehand line work $[35,22,16]$. This exercise promotes accurate and fluid freehand line work and is common warm-up and practice exercise in the first few weeks of a design sketching course.

We "gamified" the exercise by providing real-time feedback in the form of deviation lines in red. Feedback is important in gamification and serious games, particularly to help players self-regulate their learning and achieve the desired learning outcomes $[3,9]$. We also include a 5-star score after 8 lines have been sketched and provide feedback on average precision, smoothness, and speed, all important aspects of design sketching $[35,22,16]$. See Figure 5 for more detail on the gamified line lessons.

\section{ZenSketch}

ZenSketch is an approach to the same connect-the-dots exercise influenced by game-based learning and serious games [53]. The feedback and game mechanics are influenced by stealth learning since it has been shown to help reduce user anxiety and increase performance [40]. Rather than sketching "lines," the player is actually focused on sketching bridges for the character. The feedback comes in the form of bonuses and

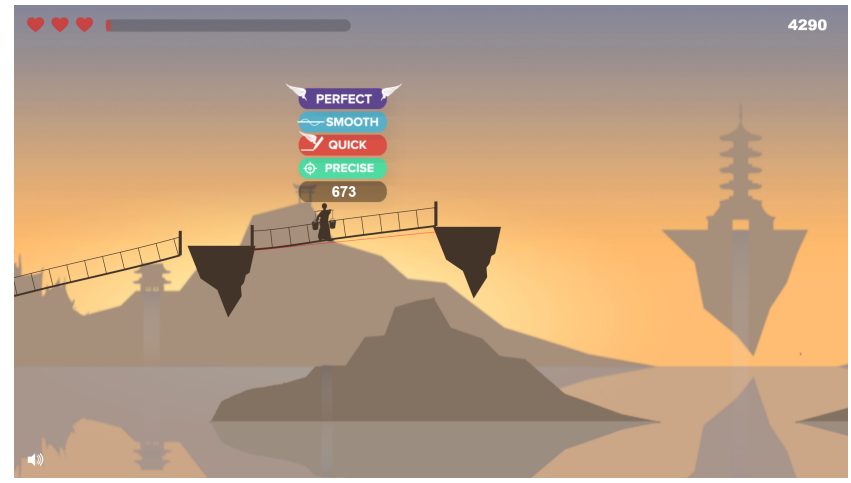

Figure 6. Screenshot of ZenSketch. The game provides real-time line feedback and bonuses during gameplay. The game also provides summative feedback and a high score, allowing for more competitive gameplay.

individual line scores, which communicate what the player is doing correctly (see Figure 6). The summative feedback is in the form of a gameover screen which shows average performance in precision, smoothness, and speed as well as providing some tips based on these values e.g., "Sketch faster to have smoother lines!"

ZenSketch also adds a leaderboard component (see Figure 9) to motivate individuals who are competitive. Utilizing high scores and leaderboards as a motivator in games has been utilized for decades [43], particularly in arcade games where players compete for "bragging rights."

\section{Methodology}

We implemented the gamified line lessons and ZenSketch in two courses for a semester-long study in Fall 2017. One course was an art course at a Giddings High School in Texas, and the other was ID1418, an introductory Industrial Design sketching course at Georgia Institute of Technology. All participants had access to both the game and the line lessons in a system called SketchTivity [51]. They had freedom to use one or the other approach, or both.

There were a total of 150 students that engaged with the systems, including 58 students from the Texas high school, and 92 students from the Georgia Tech sketching course.

We allowed the instructors to implement SketchTivity in their classes as they deemed suitable so as to not interfere with their teaching methodology. This encouraged more naturalistic gaming and resulted in data which could be described as organic. The students had access to the game and line lessons and could play them at their own leisure instead of being strictly required to play it at certain times, although the university students were encouraged by their instructors to practice periodically. The importance of this methodology was to be able to make observations on how well the approaches motivated the students.

In an effort to understand which approach students seemed to prefer, we logged lines practiced, time stamps, games played, scores, and metrics for each line including precision, smoothness, and speed data. We also conducted semi-structured interviews with three Georgia Tech students who played ZenSketch 
extensively for more specific insights on the experiences they had while playing the game over the semester. It is important to note that we did not look at overall improvement trends because this data was very asymmetrical, however we did investigate the performance of the students who played the game extensively.

\section{CASE STUDY RESULTS}

In general, both approaches were engaging and motivating to students with 72,842 lines practiced overall and the average student practicing 243 lines $( \pm 425.12)$ over the semester.

Collectively, nearly twice as many lines were practiced with the gamified line lessons $(47,842)$ versus ZenSketch $(25,002)$. This is a statistically significant difference $(\mathbf{p}=\mathbf{0 . 0 0 0 1})$ when comparing data from the two systems with a paired t-test.

The students who were highly engaged by ZenSketch were a minority. The students that played the game 10 or more times comprised only 26 of the 150 students, but accounted for nearly $64 \%$ of the 25,002 lines practiced in ZenSketch. In later sections we further investigate three of these participants.

The following results are organized in themes we found for the semester study based on analysis of the data. We did not focus on comparing activity between the two courses since the data was asymmetrical (both prototypes were used much more in the Georgia Tech sketching course where it was a requirement to practice for at least 15 minutes each week).

\section{Overall Engagement}

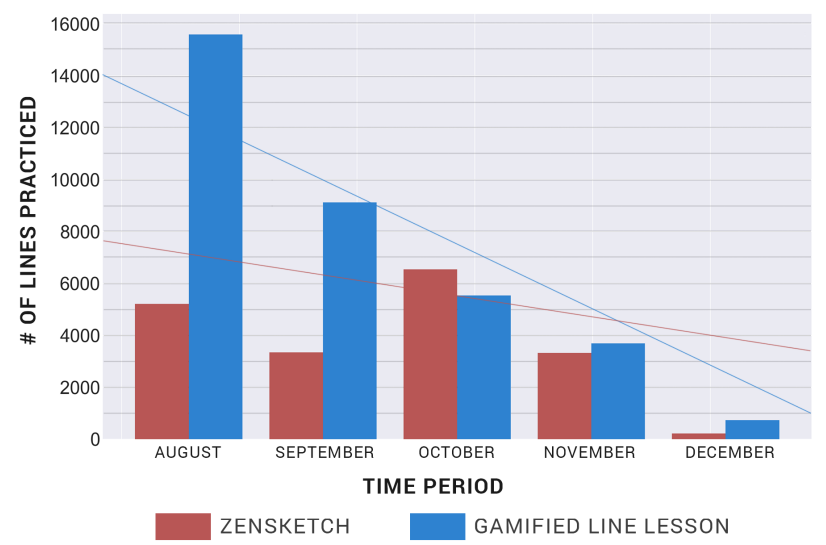

Figure 7. A histogram showing lines practiced over time for both ZenSketch and the gamified line lessons. There was a natural drop-off over time in both cases, but in October there was more lines practiced with ZenSketch. This happens to coincide with when the high school students used the system the most.

As one might expect, there was clearly initial excitement, but some natural loss of interest over the course of the semester. Figure 7 shows lines practiced from when they created an account and had access to the system.

Figure 8 shows lines practiced over the course of the semester in a bubble chart. It can be observed that participants tended to practice lines in sessions or "bursts." This is likely due to motivations to improve on their score and/or the competitive aspect of the game which is discussed in the following sections.

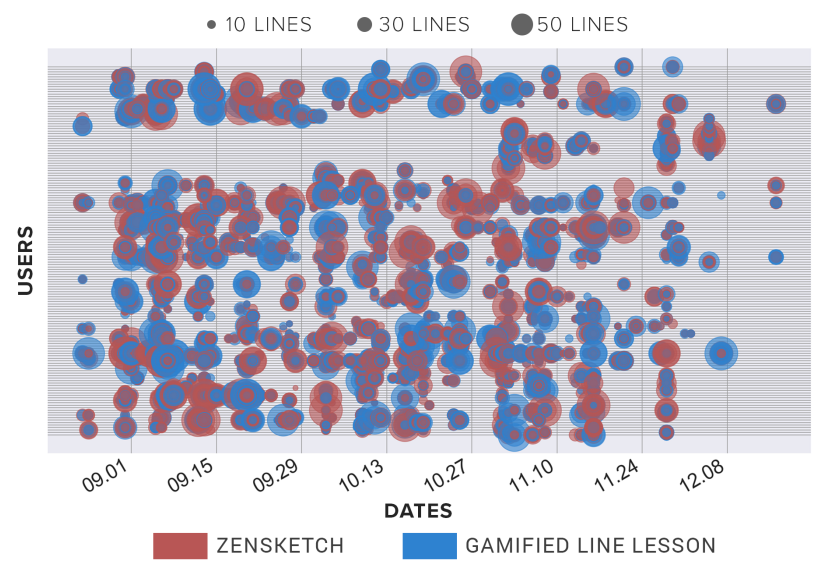

Figure 8. Game plays of all 150 users over the semester study. Each horizontal line in the chart corresponds to a user. If multiple lines were practiced in a day (sessions) we encoded that in to bubble size. It can be observed that players tended to practice lines in "bursts," likely in an effort to attain a better star rating (achievement) or a better rank on the leaderboard (competition).

\section{Competition as a Positive}

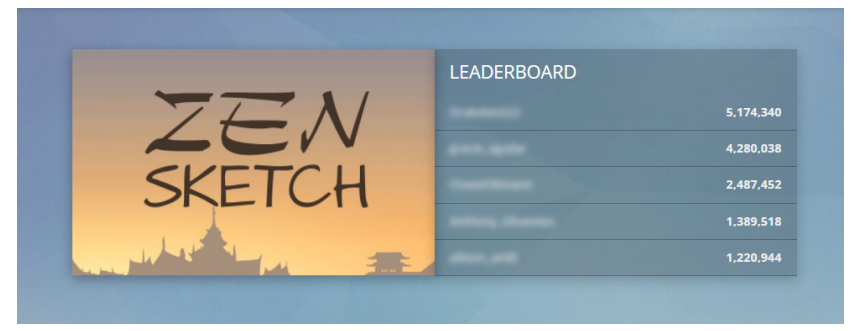

Figure 9. The leaderboard was found to be a highly motivating factor for some students, including participant CS1 who played the game 58 times in an effort to achieve the top score.

We found that the high score leaderboard component of the game (Figure 9) was a strong factor in encouraging repeated plays, particularly for participants who identified as being competitive.

Participant CS1 was an undergraduate student at Georgia Tech studying industrial design who was noteworthy for having played the game the most of any student -58 game plays in a period less than two months, practicing 1,711 lines, also the most of any student. We found that she was primarily motivated to play the game as much as she did because she found it "addictive" and was aiming for the high score in her class of 92 students.

"I found it was a pretty addictive game." "There was one particular score at the top. They had a top score and I was in $2^{\text {nd }}$ place or something. I was wanting to try to beat it. I could never beat it!"-CSI

Participant CS2 was another undergraduate student at Georgia Tech studying industrial design who played the game 14 times in her own effort to get the top score.

"...also having the scoreboard at the end where you can see oh this person in my class got like a million points, it 
gives you a goal-I have to beat them. I got more in to it when I was trying to beat other people's scores."-CS2

CS1 found the game to be much more appealing than the traditional connect-the-dots exercise common in the first few weeks of a design sketching course. She also found it more appealing than the gamified line lessons.

"I was doing a lot of the exercises but honestly was just getting kind of bored with it... And the game was just a lot of fun." - CSI

CS2 felt similarly, noting that the game made her feel more accomplished.

"Having the game definitely makes motivation easier. It makes you feel like you're actually accomplishing something instead of just like doing practice for nothing."CS2

\section{Competition as a Negative}

One participant, an undergraduate Mechanical Engineering student from Georgia Tech whom we will identify as O1, admitted to using a laptop with a track pad instead of a stylus to achieve the highest score of all participants in both studies. The study was relatively informal and encouraged the use of any number of devices, therefore we could not account for this. We had been suspicious that this participant utilized a bug in the game to achieve her score, but she openly admitted to using her track pad in the interview.

This data was thrown out, however we believed it was important to mention as an example of how extrinsic motivators such as competing with others for a high score can sometimes lead to unintended and unwanted behavior in serious games. O1 was so motivated to achieve the highest score in her class that she bypassed the intention of the game and did not cultivate improvements in her line work through using a stylus.

\section{Self-Perceived Improvement and Actual Improvement}

Both CS1 and CS2 believed that they improved their line work by playing the game over the course of the semester.

"It could still be a little tedious at times since it was just lines. But I did feel like I was a lot more confident at drawing straight lines after that."-CS2

We analyzed their data and found that CS1 kept precision and smoothness high while trending better in speed over time (Figure 10). CS2 improved in precision, but sacrificed smoothness and speed (Figure 11). This is consistent with a common "trade-off" that is made between precision and speed in motor control tasks in general $[56,38]$ and specifically in sketching [15]. People tend to sacrifice some precision in favor of speed and rapid idea generation when sketching.

This also suggests that significant ZenSketch gameplay does not necessarily correlate to a correspondingly significant improvement in line work. This may mean that only a moderate amount of gameplay can result in the desired improvement in line work that the game is designed for and further plays may have diminishing returns.

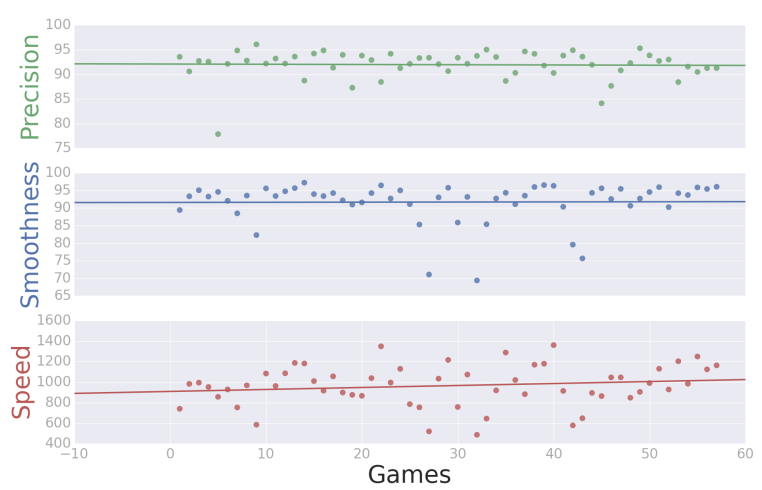

Figure 10. CS1 performance over 58 ZenSketch games

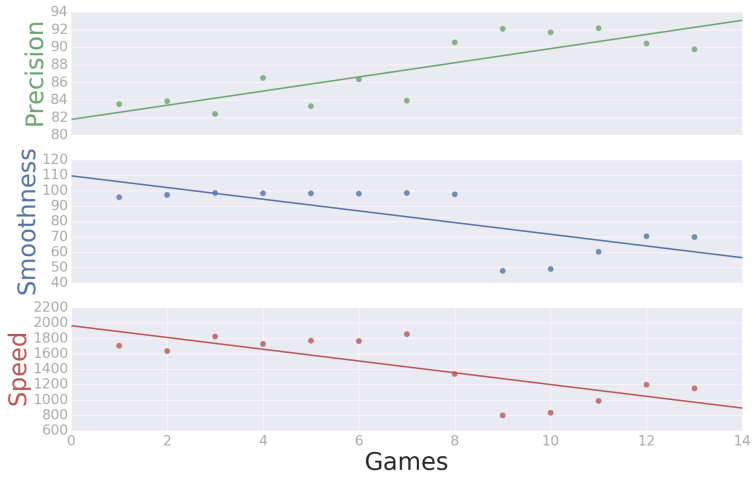

Figure 11. CS2 performance over 14 ZenSketch games

\section{Translation to paper and traditional media}

When asked if she felt like playing ZenSketch on a tablet translated to better line work on paper, CS1 responded that she believed it did.

$$
\text { “Oh yeah, yeah it definitely did-At least for lines."-CS1 }
$$

Other work in this area has shown it to be a very promising approach [21, 31, 36]. Independent of drawing on a tablet or paper, the user is cultivating the same fine motor skills, namely holding a precision instrument and moving it with increased confidence, precision, and fluidity.

\section{DISCUSSION AND FUTURE WORK}

This section includes lessons we've learned from this work, and general principles that could be useful for other researchers, educators, and technologists exploring how serious games, game-based learning, and gamification can motivate students.

\section{Diving Deeper In to Motivation}

Because of the intentionally open nature of the implementation and the organic use of our systems, it is difficult to more deeply understand student engagement and motivation from this data. Some things worth noting that influence these results:

- A greater number of lines can be practiced in a shorter time frame with the gamified line lessons. This may contribute 
to the overall trend that more lines were practiced with the line lessons.

- The university students were required to practice at least 15 minutes a week as part of a participation grade during the entire semester, whereas the high school students had more freedom to use or not use the system over a shorter period. This may have influenced them to use the tools quite differently.

- Students may have had limited access to quality devices that allow for stylus input, influencing how they engaged with the system. This has been a concern with previous studies [20].

A more controlled study in the future, and motivation scales [33] along with more interviews could determine more nuances of how these approaches are engaging and motivating students.

\section{Consistency with Our Motivation Framework}

From the lens of our motivation framework and SDT [37], the results of the case study make sense. Most of the participants were novices with little to no exposure to sketching, so many of them may have been deterred by the competitive aspect of ZenSketch. While the participants we highlighted loved the game and were very motivated by it, they were a minority among the participants from the university course. They did self-identify as being fairly confident and intermediate at sketching, so this is consistent with them being open to the competitive aspects of the game and its leaderboard, where comparison to peers is inevitable.

This does not mean the competitive aspects of ZenSketch were a poor design choice, rather that they only engaged a certain subset of people who were likely competitive by nature and/or more confident in their sketching skill. This is why we suggest that competition be used as an option for gameplay, but not as a requirement. Variety is key, and some individuals may never be drawn to competition regardless of their skill level.

\section{Utilizing Variety in Motivators}

It is well known that motivations differ greatly from student to student. Even within a medium like video games, there is a spectrum of preferences from individual to individual. Based on the results of our study, as well as recent research on rewards in games [32], we recommend researchers and game designers consider as much variety as possible when creating gamified or game-based learning solutions to motivate students. This could include adaptive systems that give the ability for students to self-select what motivates them, or select whether or not they are competitive. It could also include games that offer many different motivators within the same experience such as high scores, collectible items, cooperation with others, character growth and development, etc. It's worth noting that there is no "one-size fits all" approach and that it is highly dependent on the domain and context.

\section{Advancing Sketching Education}

In order to reap the many benefits of sketching, students must practice, and educators must consider all tools available to them to motivate their students. We believe this motivation framework can be one such tool.

We want to emphasize that this framework does not have to be limited to digital gameplay. Indeed, gameplay has been utilized in educational contexts long before the digital era. As was discussed earlier, sketch-based gameplay can include traditional pen-and-paper games like Tic-tac-toe and Hangman. Even modern educational approaches such as "sketch aerobics" utilize traditional media. The most important objective it to use sketch-based gameplay to motivate individuals of different skill levels to practice sketching, regardless of the particular medium used. Granted, digital media does not allow for certain advantages such as real-time feedback, more immersive experiences, and opportunities for remote gameplay.

We also want to emphasize that although this framework is oriented towards sketching in industrial design, many of the same principles are likely to hold true in other drawing contexts including fine art drawing [10], sketching in HCI [26], concept sketching [35], cartooning, etc. In all of these domains, students will struggle with motivation from time to time. Sketch-based gameplay can be a way to bring joy in to the learning experience while also sparking motivation. We encourage educators to experiment and researchers to share their findings.

\section{CONCLUSION}

We've presented a motivation study which describes how the motivations of individuals with different skill levels in sketching change and evolve as they improve. We've used that information to develop a motivation framework which can guide the implementation of sketch-based gameplay solutions to motivate sketching practice. We've also presented the results of a case study from the Fall 2017 school semester which involved the implementation of two different sketch-based gameplay approaches in a Texas high school art course and Georgia Tech design sketching course. The results showed both approaches were engaging, but that the gamified line lessons were more utilized overall. Many of the lines practiced with ZenSketch were from a minority of participants who were drawn to its competitive aspects. This shows some consistency with our framework, and we've offered other insights from the study that we hope can help others invested in utilizing gameplay for motivation.

\section{ACKNOWLEDGEMENTS}

We would like to thank the instructors who graciously implemented our prototypes in their classrooms. This material is based in part upon work supported by the National Science Foundation under Grant Numbers 1441331 and 1441291 and by Microsoft (Surface Hub Grant). Any opinions, findings, and conclusions or recommendations expressed in this material are those of the authors and do not necessarily reflect the views of the National Science Foundation nor Microsoft. 


\section{REFERENCES}

[1] Albert Bandura. 1997. Self-Efficacy: The Exercise of Control ( $1^{\text {st }}$ ed.). Worth Publishers, New York, New York, USA. ISBN=9780716728504.

[2] Richard Bartle. 1996. Hearts, Clubs, Diamonds, Spades: Players who Suit MUDs. Journal of MUD Research 1, 1 (1996), 19. http://mud.co.uk/richard/hcds.htm

[3] Deborah L Butler and Philip H Winne. 1995. Feedback and Self-Regulated Learning: A Theoretical Synthesis. Review of educational research 65, 3 (1995), 245-281. DOI : http://dx.doi.org/10.3102/00346543065003245

[4] Bill Buxton. 2010. Sketching User Experiences: Getting the Design Right and the Right Design. Morgan kaufmann, Burlington, Massachusetts. ISBN=9780123740373.

[5] Kathy Charmaz. 2014. Constructing Grounded Theory: A Practical Guide through Qualitative Analysis. Sage, London, United Kingdom. ISBN=978-0857029140.

[6] Yu-kai Chou. 2015. Actionable Gamification-Beyond Points, Badges, and Leaderboards. In Gamification-Engaging your workforce. ArkGroup, London, United Kingdom. ISBN=1511744049.

[7] Karen Ernst daSilva. 2001. Drawing on Experience: Connecting Art and Language. Primary Voices K-6 10, 2 (2001), 2-9. ERIC=EJ634470.

[8] Daniel Dixon, Manoj Prasad, and Tracy Hammond. 2010. iCanDraw: Using Sketch Recognition and Corrective Feedback to Assist a User in Drawing Human Faces. In Proceedings of the SIGCHI Conference on Human Factors in Computing Systems (CHI 10). ACM, New York, New York, USA, 897-906. DOI : http://dx.doi.org/10.1145/1753326.1753459

[9] Ian Dunwell, Sara de Freitas, and Steve Jarvis. 2011. Four-Dimensional Consideration of Feedback in Serious Games. In Digital games and learning. Continuum Publishing, Perth, Australia, 42-62. http://hdl.handle.net/20.500.11937/7496

[10] Betty Edwards. 1999. The New Drawing on the Right Side of the Brain ( $2^{\text {nd }}$ ed.). Tarcher, New York, New York, USA. ISBN=978-1585429219.

[11] Jennifer Fernquist, Tovi Grossman, and George Fitzmaurice. 2011. Sketch-Sketch Revolution: An Engaging Tutorial System for Guided Sketching and Application Learning. In Proceedings of the $24^{\text {th }}$ annual ACM Symposium on User Interface Software and Technology. ACM, Santa Barbara, California, USA, 373-382. http://www. jstor.org/stable/20715757

[12] Kathleen A Flannery and Malcolm W Watson. 1991. Perceived Competence in Drawing During the Middle Childhood Years. Visual Arts Research 17, 2 (1991), 66-71. https://www. jstor.org/stable/20715757

[13] Barney G Glaser. 1965. The Constant Comparative Method of Qualitative Analysis. Social Problems 12, 4 (1965), 436-445. DOI :

http://dx.doi.org/10.2307/798843
[14] Barney G Glaser and Anselm Strauss. 1978. Strauss (1967): The Discovery of Grounded Theory: Strategies for Qualitative Research. London: Wiedenfeld and Nicholson 81 (1978), 86. ISBN=978-0202302607.

[15] Tracy Hammond, Shalini Priya Ashok Kumar, Matthew Runyon, Josh Cherian, Blake Williford, Swarna Keshavabhotla, Stephanie Valentine, Wayne Li, and Julie Linsey. 2018. Its Not Just about Accuracy: Metrics That Matter When Modeling Expert Sketching Ability. ACM Transactions on Interactive Intelligent Systems (TiiS) 8, 3 (2018), 19. DOI : http://dx.doi .org/10.1145/3181673

[16] Jay D. Helsel. 1979. Reading Engineering Drawings Through Conceptual Sketching ( $1^{\text {st }}$ ed.). Glencoe/Mcgraw-Hill, Columbus, Ohio, USA. ISBN=978-0070280311.

[17] James W. Hennessey, Han Liu, Holger Winnemöller, Mira Dontcheva, and Niloy J. Mitra. 2017. How2Sketch: Generating Easy-to-Follow Tutorials for Sketching 3D Objects. In Proceedings of the $21^{\text {st }}$ ACM SIGGRAPH Symposium on Interactive 3D Graphics and Games (I3D '17). ACM, New York, NY, USA, Article 8, 11 pages. DOI : http://dx.doi .org/10.1145/3023368.3023371

[18] Ioanna Iacovides, James Aczel, Eileen Scanlon, Josie Taylor, and Will Woods. 2011. Motivation, Engagement and Learning through Digital Games. International Journal of Virtual and Personal Learning Environments (IJVPLE) 2(2) (2011), 1-16. DOI :

http://dx.doi.org/10.4018/jvple.2011040101

[19] Gabe Johnson and Ellen Yi-Luen Do. 2009. Games for Sketch Data Collection. In Proceedings of the $6^{\text {th }}$ Eurographics Symposium on Sketch-Based Interfaces and Modeling. ACM, New Orleans, LA, 117-123. DOI: http://dx. doi .org/10.1145/1572741.1572762

[20] Swarna Keshavabhotla, Blake Williford, Ethan Hilton, Paul Taele, Wayne Li, Julie Linsey, and Tracy Hammond. 2017. Conquering the Cube: Learning to Sketch Primitives in Perspective with an Intelligent Tutoring System. In SBIM '17 Proceedings of the Symposium on Sketch-Based Interfaces and Modeling, Vol. 2. ACM, Los Angeles, CA, USA, 1-11. DOI : http://dx.doi.org/10.1145/3092907.3092911

[21] Hong-Hoe Kim, Stephanie Valentine, Paul Taele, and Tracy Hammond. 2015. EasySketch: A Sketch-Based Fine Motor Skill Recognizing Educational Interface for Children Emerging Technology Research Strand. In The Impact of Pen and Touch Technology on Education, Tracy Hammond, Stephanie Valentine, Aaron Adler, and Mark Payton (Eds.). Springer Publishing Company, Incorporated, Switzerland, Chapter 4, 35-46. DOI : http://dx.doi.org/10.1007/978-3-319-15594-4_4

[22] Joseph A. Koncelik and Kevin Reeder. 2008. Conceptual Drawing ( $1^{\text {st }}$ ed.). Course Technology, Boston, Massachusetts, USA. ISBN=978-1418080976. 
[23] Christina Lassheikki, Iina Nokelainen, Yuanqi Shan, Maximus Kaos, Martti Raevaara, Heikka Valja, and Perttu Hämäläinen. 2018. Fair and Encouraging_-Developing an Automatic Assessment System in Aalto Drawing. In Proceedings of the 2018 Annual Symposium on Computer-Human Interaction in Play Companion Extended Abstracts. ACM, Melbourne, VIC, Australia, 337-344. DOI :

http://dx.doi.org/10.1145/3270316.3270318

[24] Edith Law and Luis Von Ahn. 2009. Input-Agreement: A New Mechanism for Collecting Data using Human Computation Games. In Proceedings of the SIGCHI Conference on Human Factors in Computing Systems. ACM, Boston, Massachusetts, USA, 1197-1206. DOI : http://dx.doi.org/10.1145/1518701.1518881

[25] Yong Jae Lee, C Lawrence Zitnick, and Michael F Cohen. 2011. Shadowdraw: Real-Time User Guidance for Freehand Drawing. In ACM Transactions on Graphics (TOG), Vol. 30. ACM, New York, New York, USA, 27. DOI :

http://dx.doi.org/10.1145/2010324. 1964922

[26] Makayla Lewis, Miriam Sturdee, Jason Alexander, Jelle Van Dijk, Majken Kirkegård Rasmussen, and Thuong Hoang. 2017. SketchingDIS: Hand-Drawn Sketching in HCI. In Proceedings of the 2017 ACM Conference Companion Publication on Designing Interactive Systems. ACM, Edinburgh, 356-359. DOI : http://dx.doi.org/10.1145/3064857.3064863

[27] Thomas W Malone. 1981. Toward a Theory of Intrinsically Motivating Instruction. Cognitive science 5, 4 (1981), 333-369. DOI :

http://dx.doi.org/10.1207/s15516709cog0504_2

[28] Scott Nicholson. 2013. Two Paths to Motivation through Game Design Elements: Reward-Based Gamification and Meaningful Gamification. In iConference 2013. iSchools, Inc., Fort Worth, Texas, USA, 671-672. http://hdl.handle.net/2142/42082

[29] Michael I Norton, Daniel Mochon, and Dan Ariely. 2012. The IKEA Effect: When Labor Leads to Love. Journal of Consumer Psychology 22, 3 (2012), 453-460. DOI : http://dx.doi.org/10.1016/j.jcps.2011.08.002

[30] OMGPop and Zynga. 2012. DrawSomething. Game [Mobile]. (6 February 2012). omgpop.com/drawsomething

[31] Brandon Paulson, Brian Eoff, Aaron Wolin, Joshua Johnston, and Tracy Hammond. 2008. Sketch-Based Educational Games: Drawing Kids Away from Traditional Interfaces. In Proceedings of the $7^{\text {th }}$ international conference on Interaction Design and Children (IDC). ACM, Chicago, IL, USA, 133-136. ISBN=978-1-59593-994-4.

[32] Cody Phillips, Daniel Johnson, Madison Klarkowski, Melanie Jade White, and Leanne Hides. 2018. The Impact of Rewards and Trait Reward Responsiveness on Player Motivation. In Proceedings of the 2018 Annual
Symposium on Computer-Human Interaction in Play. ACM, Melbourne, VIC, Australia, 393-404. DOI :

http://dx.doi.org/10.1145/3242671.3242713

[33] Paul R Pintrich and others. 1991. A Manual for the Use of the Motivated Strategies for Learning Questionnaire (MSLQ). National Center for Research to Improve Postsecondary Teaching and Learning Project on Instructional Processes and Educational Outcomes OERI-86-0010, Article NCRIPTAL-91-B-004 (1991), 76 pages. ERIC Number: ED338122.

[34] Robert Angel. 1985. Pictionary. Game [Board Game]. (1985). mattel.com

[35] Scott Robertson and Thomas Bertling. 2013. How to Draw: Drawing and Sketching Objects and Environments from your Imagination ( $1^{\text {st }}$ ed.). Design Studio Press, Culver City, California, USA. ISBN=978-1933492735.

[36] James C Rosser, Paul J Lynch, Laurie Cuddihy, Douglas A Gentile, Jonathan Klonsky, and Ronald Merrell. 2007. The Impact of Video Games on Training Surgeons in the $21^{\text {st }}$ Century. Archives of surgery 142, 2 (2007), 181-186. DOI :

http://dx.doi .org/10.1001/archsurg.142.2.181

[37] Richard M Ryan and Edward L Deci. 2000. Self-Determination Theory and the Facilitation of Intrinsic Motivation, Social Development, and Well-Being. American Psychologist 55, 1 (2000), 68. DOI : http://dx.doi.org/10.1037/0003-066X.55.1.68

[38] Richard A. Schmidt, David E. Sherwood, Howard N. Zelaznik, and Bernard J. Leikind. 1985. Speed-Accuracy Trade-offs in Motor Behavior: Theories of Impulse Variability. In Motor Behavior: Programming, Control, and Acquisition, Herbert Heuer, Uwe Kleinbeck, and Klaus-Helmut Schmidt (Eds.). Springer Berlin Heidelberg, Berlin, Heidelberg, 79-123. DOI : http://dx.doi .org/10.1007/978-3-642-69749-4_3

[39] Kathy G. Short, Gloria Kauffman, and Leslie H. Kahn. 2000. "I Just Need To Draw": Responding to Literature across Multiple Sign Systems. The Reading Teacher 54, 2 (Oct 2000), 160-171. ISSN-0034-0561.

[40] Valerie J Shute. 2011. Stealth Assessment in Computer-based Games to Support Learning. Computer Games and Instruction 55, 2 (2011), 503-524. ISBN=978-1617354083.

[41] Jaakko Stenros. 2015. Playfulness, Play, and Games: A Constructionist Ludology Approach. Master's thesis. Tampere University, Finland. http://urn. fi/URN : ISBN : 978-951-44-9788-9

[42] Miriam Sturdee, Samuel Mann, and Sheelagh Carpendale. 2019. Sketching Sustainability in Computing. In Proceedings of the 2019 on Creativity and Cognition. ACM, San Diego, California, USA, 29-40. DOI : http://dx.doi.org/10.1145/3325480.3325481 
[43] Zachary O Toups, Andruid Kerne, and William Hamilton. 2009. Motivating Play Through Score. In In ACM Computer Human Interaction Workshop on Engagement by Design. ACM, Boston, Massachusetts, USA, Article ACM 978-1-60558-247-4/08/04, 4 pages. http://citeseerx.ist.psu.edu/viewdoc/summary?doi=10. 1.1.410.4356

[44] Chau Thai Truong, Duy-Hung Nguyen-Huynh, and Minh-Triet Tran. 2014. How Do We Teach Young Children New Concepts via Sketching?. In Distributed, Ambient, and Pervasive Interactions, Norbert Streitz and Panos Markopoulos (Eds.). Springer International Publishing, Limenas Chersonisou, Greece, 292-303. DOI : http://dx.doi.org/10.1007/978-3-319-07788-8_28

[45] Peter Van Sommers. 1984. Drawing and Cognition: Descriptive and Experimental Studies of Graphic Production Processes. Cambridge University Press, New York, New York, USA. DOI : http://dx.doi.org/10.1017/СВ09780511897672

[46] Peter van Sommers. 1989. A System for Drawing and Drawing-Related Neuropsychology. Cognitive Neuropsychology 6, 2 (Mar 1989), 117-164. DOI : http://dx.doi .org/10.1080/02643298908253416

[47] Francisco Vides. 2012. TAYouKi: A Sketch-Based Tutoring System for Young Kids. MS Master's Thesis. Texas A\&M University (TAMU), College Station, TX, USA. Advisor: Tracy Hammond, http://hdl . handle.net/1969.1/ETD-TAMU-2012-08-11497.

[48] Francisco Vides, Paul Taele, Hong-Hoe (Ayden) Kim, and Tracy Hammond. 2012. Intelligent Feedback for Kids Using Sketch Recognition. In ACM SIGCHI 2012 Conference on Human Factors in Computing Systems Workshop on Educational Interfaces, Software, and Technology. ACM, Austin, Texas, USA, Article 15, 4 pages. http://smarttech. com/eist2012

[49] Rajinder Wasson, David Mould, Robert Biddle, and Cristina S. Martinez. 2013. A Sketching Game for Art History Instruction. In Proceedings of the International Symposium on Sketch-Based Interfaces and Modeling (SBIM '13). ACM, New York, NY, USA, 23-31. D0I : http://dx.doi.org/10.1145/2487381.2487384

[50] Nicola Jane Whitton. 2007. An Investigation into the Potential of Collaborative Computer Game-Based
Learning in Higher Education. Ph.D. Dissertation. Edinburgh Napier University.

http://researchrepository .napier .ac.uk/id/eprint/4281

[51] Blake Williford. 2017. SketchTivity: Improving Creativity by Learning Sketching with an Intelligent Tutoring System. In Proceedings of the 2017 ACM SIGCHI Conference on Creativity and Cognition. ACM, Singapore, 477-483. DOI :

http://dx.doi.org/10.1145/3059454.3078695

[52] Blake Williford, Abhay Doke, Michel Pahud, Ken Hinckley, and Tracy Hammond. 2019. DrawMyPhoto: Assisting Novices in Drawing from Photographs. In Proceedings of the 2019 on Creativity and Cognition. ACM, San Diego, California, USA, 198-209. DOI : http://dx.doi.org/10.1145/3325480.3325507

[53] Blake Williford, Matthew Runyon, Adil Hamid Malla, Wayne Li, Julie Linsey, and Tracy Hammond. 2017. ZenSketch: A Sketch-based Game For Improving Line Work. In Extended Abstracts Publication of the Annual Symposium on Computer-Human Interaction in Play. ACM, Amsterdam, 591-598. DOI : http://dx.doi . org/10.1145/3130859.3130861

[54] Pieter Wouters, Christof Van Nimwegen, Herre Van Oostendorp, and Erik D Van Der Spek. 2013. A Meta-Analysis of the Cognitive and Motivational Effects of Serious Games. Journal of Educational Psychology 105, 2 (2013), 249. https:

//psycnet.apa.org/doiLanding?doi=10.1037\%2Fa0031311

[55] Jun Xie, Aaron Hertzmann, Wilmot Li, and Holger Winnemöller. 2014. PortraitSketch: Face Sketching Assistance for Novices. In Proceedings of the $27^{\text {th }}$ Annual ACM Symposium on User Interface Software and Technology (UIST'14). ACM, New York, New York, USA, 407-417. DOI : http://dx.doi.org/10.1145/2642918.2647399

[56] Shumin Zhai, Jing Kong, and Xiangshi Ren. 2004. Speed-Accuracy Tradeoff in Fitts' Law Tasks-On the Equivalency of Actual and Nominal Pointing Precision. International Journal of Human-Computer Studies 61, 6 (2004), 823-856. DOI : http://dx.doi.org/10.1016/j.ijhcs.2004.09.007 